\title{
Long-term partial reinforcement extinction effect and long-term partial punishment effect in a one-trial-a-day paradigm
}

\author{
ANNE SHEMER and JORAM FELDON \\ Tel Aviv University, Tel Aviv, Israel
}

\begin{abstract}
Two experiments were run to demonstrate the presence of a partial reinforcement extinction effect (PREE) and a partial punishment effect (PPE) 4 weeks after training in a 1-trial/day procedure. In the PREE paradigm, two groups of animals were trained to run a straight alley for food reward; one group was rewarded on every trial (CRF), whereas the other was rewarded on only $50 \%$ of the trials (PRF). In the test phase, extinction, no reward was present on any trial. Four weeks after the end of acquisition, and subsequent to 7 days of CRF retraining, PRF animals showed greater resistance to extinction than did their CRF controls. The PPE paradigm resembled the PREE paradigm in all aspects, except that mild electric footshock, together with the food reward, was used instead of nonreward on $50 \%$ of the acquisition trials. In the test phase, all animals were shocked and given a food reward on every trial. Four weeks after the end of acquisition and retraining, the animals with previous experience of shock in acquisition showed greater resistance to punishment than did their controls.
\end{abstract}

The aim of the present two experiments was to explore the long-term characteristics of the partial reinforcement extinction effect (PREE) and the partial punishment effect (PPE). The PREE consists of increased resistance to extinction shown by animals trained on a random partial reinforcement (PRF) schedule relative to animals trained on a continuous reinforcement (CRF) schedule (see Amsel, 1958, Lewis, 1960, and Mackintosh, 1974, for reviews). The PPE refers to the increased resistance to punishment exhibited by animals trained on a partial punishment (PP) schedule compared with that exhibited by their CRF controls (Banks, 1966; Miller, 1960).

In the latter procedure, two groups of animals, CRF and $\mathrm{PP}$, are trained to run a straight alley. Both groups receive food reinforcement on every trial. In addition, the PP group is exposed to gradually increasing levels of mild electric shock on $50 \%$ of the trials. In the test, or punishment, phase of the experiment, both groups receive reward together with shock on every trial. The shock intensity is set at the level reached by the PP animals at the end of training. The PPE refers to the finding that the PP animals continue running for the reward faster and longer than their CRF controls.

The increased resistance to punishment and extinction obtained in the PPE and PREE paradigms, respectively, has been regarded as an example of the develop-

This study was supported by grants from the Israel Academy of Sciences (Basic Research Fund) and the Ministry of Health (Chief Scientist's Office). J. Feldon is a Bat-Sheva Fellow. The authors' mailing address is: Department of Psychology, Tel Aviv University, Tel Aviv, Israel. ment of behavioral tolerance to stress (Gray, Davis, Owen, Feldon, \& Boarder, 1981). This approach argues that, during training, the PP and PRF animals learn to overcome the inhibitory effects of aversive events. With regard to PREE, Amsel (1962) claimed that the mechanism underlying the increased resistance to extinction is one of counterconditioning, whereby the environmental stimuli that had been associated with emotional responses such as frustration become cues for the continued performance of the learned response. In line with these accounts are experimental demonstrations of transfer of increased resistance from PP training to extinction and from PRF training to punishment (Brown \& Wagner, 1964).

Within the theoretical framework that emphasizes the development of behavioral tolerance as a consequence of PRF and PP training, it is important to establish their long-term effects, that is, the extent to which the beneficial effects of exposure to stress persist over time. In this respect, PREE has been studied to a much greater extent than PPE (for review, see Mackintosh, 1974). Thus, PREE has been demonstrated after relatively long time intervals between the acquisition and test stages of an experiment. These findings were obtained using a multitrial procedure, in which animals were run for several trials each day for a number of days (Chen \& Amsel, 1975; Traupman, Wong, \& Amsel, 1971). However, long-term PREE has not been shown with a 1-trial/day procedure, and, to the best of our knowledge, there is no evidence of a long-term PPE in either the multitrial or the 1-trial/day procedure.

The reason for focusing on the long-term effects of the PREE and the PPE in the 1-trial/day procedure is 
that this procedure has been used extensively in psychopharmacological and physiological research, particularly in investigations of the effects of minor tranquilizers (Davis, Brookes, Gray, \& Rawlins, 1981; Feldon \& Gray, 1981a) and lesions to the septohippocampal system (Feldon \& Gray, 1979). This research has demonstrated that certain treatments, such as septal lesions and benzodiazepine administration, affect differentially the PREE at 1 trial/day as compared with the multitrial procedure, and has led to theoretical suggestions about the role of the septohippocampal system in the development of behavioral tolerance to stress (Gray, Feldon, Rawlins, Owen, \& McNaughton, 1978), as well as about the manner in which this system might mediate the effects of minor tranquilizers on the PREE (Feldon \& Gray, 1981b). Both the PPE and PREE paradigms have been suggested as particularly suitable models of anxiety for studying the behavioral effects of anxiolytics (Gray et al., 1981; Iversen, 1980).

The establishment of the long-term characteristics of the PPE and PREE would enhance considerably the usefulness of those models in the investigation of the above-mentioned physiological and pharmacological phenomena.

In addition, the use of these paradigms as psychopharmacological tools has encountered some difficulties due to problems of state-dependent learning (Overton, 1966). State-dependent learning refers to the fact that, in an experimental procedure involving two stages, the transfer from the acquisition to the test phase of the experiment coincides with a change in drug state. Consequently, the observed effect of a drug might reflect either a direct action of the drug on the behavioral response in acquisition or the change in stimulus condition from drug state to no-drug state. Indeed, in a number of studies (Feldon \& Gray, 1981a; Gray, 1969; Ison \& Pennes, 1969), performance deficits in the test phase have occurred merely as a result of the change in drug state. The temporal separation of the acquisition and test phases would allow an evaluation of the drug effects uncontaminated by the simultaneous change in drug state.

Experiment 1 of the present study employed the PREE paradigm, and Experiment 2 used the PPE paradigm. In both experiments, a 1-trial/day procedure was employed, and the stages of acquisition and test were separated by 4 weeks.

\section{EXPERIMENT 1}

\section{Method}

Subjects. The subjects were 12 male Wistar rats approximately 90 days old. They were housed four to a cage under a 12-h reversed light/dark cycle. A 23-h food-deprivation schedule was introduced 2 weeks before the commencement of training and was maintained throughout the experiment. Water was available ad lib in the home cage.

Apparatus. The runway consisted of a straight alley made of transparent Perspex; black rubber curtains covered the sides. The alley was $140 \mathrm{~cm}$ long, $15 \mathrm{~cm}$ wide, and $35 \mathrm{~cm}$ high, with a startbox (20 cm long) and a goalbox ( $20 \mathrm{~cm}$ long) separated by a run section (100 cm long). The floor consisted of a metal grid composed of equally spaced rods. The startbox door, which opened downward, was made of transparent Plexiglas. The door was operated by a solenoid controlled by a push button. The goalbox door was made of metal; it opened vertically and was operated by hand. The food pellets were placed in a recessed compartment $40 \mathrm{~cm}$ wide and $2.5 \mathrm{~cm}$ deep at the far side of the goalbox. There were three visible light photobeams and photocells, the first one $2 \mathrm{~cm}$ beyond the startbox, the second one $2 \mathrm{~cm}$ before the goal section, and the third one within the goalbox. The latter was interrupted when the rat contacted the food compartment. The photobeams operated three electronic timers, accurate to $0.01 \mathrm{sec}$. The first one timed the start section (from the opening of the start door to the first photobeam), the second one, the run section (from the first to the second photobeam), and the third one, the goal section (from the second to the third photobeam). Food was placed manually in the goalbox compartment prior to each rewarded trial. Each reward consisted of 20 45-mg Campden Instruments food pellets.

Procedure. The animals were divided randomly into two groups-PRF and CRF. All subjects were handled for approximately $30 \mathrm{sec} /$ day for 14 days. Following this period, all animals received 2 days of pretraining. On the 1 st day, the animals were placed in the alley in groups of four for $20 \mathrm{~min}$, with food pellets available in the goalbox compartment. On the 2nd day, the animals were placed in the alley in pairs for $10 \mathrm{~min}$, again with food pellets available. On the next day, training, consisting of 1 trial/day for 16 days, was initiated. On each day, the animals were placed in the start section, and the three time measurements, for the start, run, and goal sections, were obtained for each of them. The CRF group received a reward on every trial. The PRF group received a reward on only $50 \%$ of the trials on a quasi-random schedule of RRRNNRNNRNNRNNRR, where $R$ represents a rewarded trial and $N$ represents a nonrewarded trial. At the end of 16 days, the animals were given 3 weeks of rest in their home cages. Following this period, both groups were given 7 days of CRF training with 1 trial/day. At the end of the 7 days, extinction began and continued for 10 days. During extinction, no rewards were given.

The data were transformed into reciprocals to allow the use of analysis of variance (ANOVA). ANOVAs were performed for the acquisition, reacquisition, and extinction phases. For each phase, start, run, and goal data were analyzed separately. Each analysis included one main factor of reinforcement type (PRF vs. CRF) and a repeated measurements factor of days.

\section{Results and Discussion}

Acquisition. Analysis of the data revealed no significant effects in the start and run sections of the alley. As can be seen in Figure 1, for the goal section, the PRF group tended to be slower in acquisition. This effect approached significance, as reflected by the reinforcement $x$ days interaction $[F(15,150)=1.64, p<.07]$.

Reacquisition. No significant effects in any section of the alley were revealed in the analysis of the reacquisition data. At the beginning of the 7 days of CRF training, all animals were considerably slower than they had been at the end of acquisition; by the end of retraining, however, the PRF animals ran as fast as their CRF controls.

Test. The analysis of the goal-section data revealed a significant main effect of reinforcement $[F(1,10)=$ 5.04, $\mathrm{p}<.04]$ and a significant reinforcement $\mathrm{x}$ days interaction $[F(9,90)=2.41, p<.01]$. As can be seen 

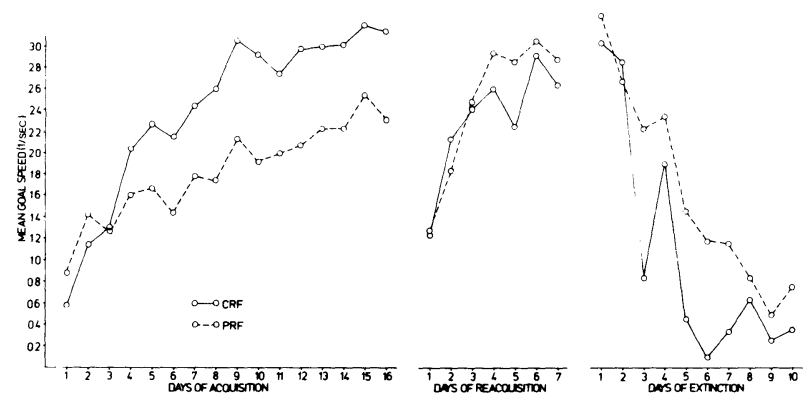

Figure 1. Course of acquisition, reacquisition, and extinction in the goal section of the alley. CRF-continuous reinforcement; PRF-partial reinforcement.

in Figure 1, these results were due to the fact that, starting from the 3rd day of extinction, the CRF animals began to slow down considerably more than did the PRF group. This is a clear PREE. A similar trend, although one that did not reach significance, was observed in the start and run sections of the alley.

It may be concluded that, after a 4-week delay in a 1-trial/day procedure, the PREE was clearly present, with the PRF group showing greater resistance to extinction than did their CRF controls.

With regard to the acquisition phase, it is interesting to note that the trend of the PRF animals to perform more slowly than their CRF controls disappeared and even slightly reversed during reacquisition. This result might be related to findings that show that animals trained under more demanding conditions tend to perform more vigorously on a subsequent task (Eisenberger, Carlson, Guile, \& Shapiro, 1979; Eisenberger, Terborg, \& Carlson, 1979).

\section{EXPERIMENT 2}

\section{Method}

Subjects. There were 15 subjects. Specifications were the same as in Experiment 1.

Apparatus. The apparatus was the same as that in Experiment 1 , except that, on punished trials, the breaking of the final photobeam automatically delivered a scrambled 0.6 -sec shock from a Lafayette shock source and scrambler to the grid floor of the goalbox.

Procedure. The subjects were divided randomly into two groups: PP (eight subjects) and CRF (seven subjects). The general procedure was the same as that in Experiment 1, except that the PRF group was replaced by the PP group and 12 acquisition trials were run. The CRF group was run exactly as that in Experiment 1. The PP animals were rewarded with food on every trial, but were exposed to gradually increasing footshock together with the food reward in the goal section. Shock was presented $0.01 \mathrm{sec}$ after the third photobeam had been broken, the signal that the animal was inside the goal section, where the food pellets were available. The shock was presented on a $50 \%$ quasi-random schedule: RRRPPRPPRPPR, where $\mathrm{R}$ represents a rewarded trial and $P$ represents a punished trial with both reward and shock. For the first two punished trials, the shock level was $0.15 \mathrm{~mA}$, for the third and fourth punished trials, the level was $0.25 \mathrm{~mA}$, and for the last two trials, the level was
$0.3 \mathrm{~mA}$. At the end of acquisition, the animals were given a 3-week rest period, reacquisition training, and then test.

In the test, or punishment stage, all animals were given a $0.3-\mathrm{mA}$ 0.6-sec shock in the goal section, together with the food reward, on every trial. The test lasted 11 days. The data were analyzed using the same procedures as those in Experiment 1 .

\section{Results and Discussion}

Acquisition. There were no significant effects in the analysis of the acquisition data. As can be seen in Figure 2, the PP animals acquired the response slightly slower than the CRF controls; however, by the end of acquisition, the two groups were identical in their running speeds.

Reacquisition. There were no significant differences in any of the alley sections.

Test. In all three sections of the alley, the PP animals ran faster than their CRF controls. This was substantiated in the start section by the significant reinforcement $x$ days interaction $[F(10,130)=2.34, p<.01]$. In the run section, this effect approached significance in the main effect of reinforcement $[F(1,13)=3.27, p<.09]$ and in the reinforcement $x$ days interaction $[F(10,130)$ $=1.69, \mathrm{p}<.08]$. The results of the goal section are presented in Figure 2. As can be seen in Figure 2, from the 3rd day onward, the two groups diverged, the PP animals continuing to run and the CRF animals slowing down considerably. This was supported by the significant main effect of reinforcement $[F(1,13)=4.43$, $\mathrm{p}<.05]$ and by the significant interaction of reinforcement $x$ days $[F(10,130)=2.72, p<.004]$.

It can be concluded that, after a delay of 4 weeks between the acquisition and test phases, a clear PPE was obtained, the PP group showing greater resistance to punishment than their CRF controls.

\section{GENERAL DISCUSSION}

The presence of a PREE and a PPE 4 weeks after training demonstrates that, with relatively limited experience with aversive events (eight nonrewarded trials or six exposures to mild footshock), animals can learn to overcome the disruptive effects of aversive stimuli and show the effects of such experience

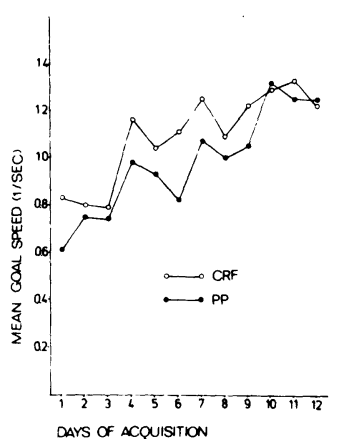

DAYS Of ACOUISITION
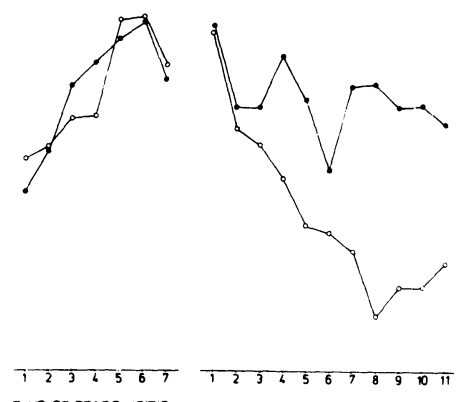

DAYS OF REACQUISITION

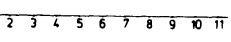

DASS OF TEST
Figure 2. Course of acquisition, reacquisition, and punishment test in the goal section of the alley. CRF-continuous reinforcement; $\mathrm{PP}$-partial punishment. 
even weeks later. The mechanisms underlying these effects may be several, and they are not necessarily the same for the two paradigms.

For the PREE phenomenon, two alternative explanations have been proposed: Capaldi (1967) emphasized the intertrial mechanism, whereby the animal comes to use the memory traces of nonreward as cues for the continued performance of the partially rewarded response. In contrast, Amsel (1962) stressed the intratrial mechanism, whereby the environmental stimuli, previously associated with emotional responses such as fear or frustration, become, by a process of counterconditioning, cues for the performance of the learned response. It appears (Gray, 1975; Mackintosh, 1974) that both mechanisms play a part in the PREE, with the specific conditions employed maximizing the intertrial or intratrial route. Whereas short intertrial intervals and small rewards are likely to give rise to the intertrial mechanism, long intertrial intervals and large rewards are likely to promote intratrial mechanisms. Since the latter conditions were employed in the present study, it may be assumed that the long-term effects obtained with PRF training were mediated by the counterconditioning of frustration. This assumption is also supported by PREE studies that have used antianxiety drugs. Anxiolytics are assumed to act on the emotional responses to aversive stimuli, such as frustration, and not on the memory traces of the learning situation. Using a short intertial-interval procedure, Ziff and Capaldi (1971) reported that the PREE was not affected by anxiolytics. In contrast, in the 1-trial/day procedure, a clear drug effect was obtained (Feldon \& Gray, 1981a; Feldon, Guillamon, Gray, de Wit, \& McNaughton, 1979).

Whatever the mechanism behind these two phenomena, the long-term effects are both interesting and useful. There remain, however, many unanswered questions: How long do the effects of increased tolerance last? To what extent are they transferable? Is there any underlying pharmacological or physiological change? We hope to address some of these questions in the future.

\section{REFERENCES}

Amsel, A. (1958). The role of frustrative non-reward in noncontinuous reward situations. Psychological Bulletin, 55, 102-119.

Amsel, A. (1962). Frustrative non-reward in partial reinforcement and discrimination learning: Some recent history and a theoretical extension. Psychological Review, 69, 306-328.

Banks, R. K. (1966). Persistence to continuous punishment following intermittent punishment training. Journal of Experimental Psychology, 71, 373-377.

Brown, R. T., \& WAgner, A. R. (1964). Resistance to punishment and extinction following training with shock or reinforcement. Journal of Experimental Psychology, 68, 503-507.

Capaldi, E. J. (1967). A sequential hypothesis of instrumental learning. In R. W. Spence \& J. T. Spence (Eds.), The psychology of learning and motivation (Vol. 1, pp. 67-156). New York: Academic Press.

Chen, J. S., \& Amsel, A. (1975). Retention and durability of persistence acquired by young and infant rats. Journal of Comparative and Physiological Psychology, 89, 238-245.

Davis, N., Brookes, S., Gray, J. A., \& Rawlins, J. (1981). Chlordiazepoxide and resistance to punishment. Quarterly Journal of Experimental Psychology, 33B, 227-239.
Eisenberger, R., Carlson, J., Guile, M., \& Shapiro, N. (1979). Transfer of effort across behaviors. Learning and Motivation, 10, 178-197.

Eisenberger, R., Terborg, R., \& Carlson, J. (1979). Transfer of persistence across reinforced behaviors. Animal Learning and Behavior, 7, 493-498.

Feldon, J., \& Gray, J. A. (1979). Effects of medial and lateral septal lesions on the partial reinforcement extinction effect at one trial a day. Quarterly Journal of Experimental Psychology, 31, 653-674.

Feldon, J., \& Gray, J. A. (1981a). The partial reinforcement extinction effect after treatment with chlordiazepoxide. Psychopharmacology, 73, 269-275.

Feldon, J., \& Gray, J. A. (1981b). The partial reinforcement extinction effect and the influence of chlordiazepoxide in septal lesioned rats. Psychopharmacology, 74, 280-289.

Feldon, J., Guillamon, A., Gray, J. A., de Wit, H., \& McNaughton, N. (1979). Sodium amylobarbitone and responses to non-reward. Quarterly Journal of Experimental Psychology, 31, 19-50.

GraY, J. A. (1969). Sodium amobarbital and effects of frustrative non-reward. Journal of Comparative and Physiological Psychology, 69, 55-64.

GraY, J. A. (1975). Elements of two-process theory of learning. London: Academic Press.

Gray, J. A., Davis, N., Owen, S., Feldon, J., \& Boarder, M. (1981). Stress tolerance: Possible neural mechanisms. In M. J. Christie \& P. G. Mellet (Eds.), Foundations of psychosomatics (pp. 153-167). Chichester, England: Wiley.

Gray, J. A., Feldon, J., Rawlins, J. N. P., Owen, S., \& McNaughton, N. (1978). The role of the septohippocampal system and its noradrenergic afferents in behavioural responses to non-reward. In E. K. Whelan (Ed.), Functions of the Septohippocampal System. Ciba Foundation Symposium (Vol. 58, pp. 275-307). Amsterdam: Elsevier.

Ison, J. R., \& Pennes, E. S. (1969). Interaction of amobarbital sodium and reinforcement schedule in determining resistance to extinction of an instrumental running response. Journal of Comparative Physiological Psychology, 68, 215-219.

IvERSEN, S. D. (1980). Animal models of anxiety and benzodiazepine actions. Arznein Forschung, 30, 862-868.

LEWIs, D. J. (1960). Partial reinforcement: A selective review of the literature since 1950. Psychological Bulletin, 57, 1-28.

Mackintosh, N. J. (1974). The psychology of animal learning. London: Academic Press.

Miller, N. E. (1960). Learning resistance to pain and fear: Effects of overlearning exposure and rewarded exposure in context. Journal of Experimental Psychology, 60, 137-145.

Overton, D. A. (1966). State dependent learning produced by depressant and atropine like drugs. Psychopharmacology, 10, 6-31.

Traupman, K. L., Wong, P. T. P., \& Amsel, A. (1971). Durability of persistence as a function of number of partially reinforced trials. Journal of Experimental Psychology, 10, 6-31.

ZifF, D. R., \& CAPALdi, E. J. (1971). Amytal and the small trial partial reinforcement effect: Stimulus properties of early trial non-rewards. Journal of Experimental Psychology, 87, 263-269.

(Manuscript received for publication July 18, 1983.) 